\title{
Qualidade de vida e estado nutricional de pacientes diagnosticadas com câncer de mama
}

\section{Quality of life and nutritional status of patients diagnosed with breast cancer \\ Calidad de vida y el estado nutricional de pacientes con el diagnóstico de cáncer de mama}

\author{
Sabrina Hodecker iD \\ Universidade Regional de Blumenau - Blumenau (SC) - Brasil \\ Luciane Coutinho de Azevedo (iD \\ Universidade Regional de Blumenau - Blumenau (SC) - Brasil
}

\begin{abstract}
RESUMO
Objetivo: Avaliar a qualidade de vida e sua relação com o estado nutricional de mulheres diagnosticadas com câncer de mama. Métodos: Estudo transversal que avaliou mulheres com diagnóstico de câncer de mama frequentadoras de um programa filantrópico de combate ao câncer, em Brusque, Santa Catarina, entre abril e junho de 2018. Adotou-se como critério de exclusão apresentar incapacidade cognitiva de responder aos questionários European Organization for Research and Treatment of Cancer (QLQ-C30 e QLQ-BR23) ou impossibilidade de coleta das medidas antropométricas para classificação do estado nutricional. O total em escores dos questionários e de suas escalas e domínios deram-se como variáveis analisadas. Resultados: Participaram 40 mulheres, sendo 16 com sobrepeso (40\%), 14 com eutrofia (35\%) e 10 com obesidade (25\%). O valor médio do QLQ-C30 apresentou-se como de 72,2 $\pm 26,3$ pontos, sem diferença entre as categorias de estado nutricional. Os domínios mais afetados da escala funcional registraram-se como: emocional, cognitivo e escala sintomas, com insônia, dor e fadiga. Mulheres com sobrepeso apresentaram piora nos domínios de ambas as escalas. Os domínios perspectivas futuras, imagem corporal, função sexual e sintomas de braço apresentaram-se mais comprometidos. Mulheres submetidas à reconstrução mamária e as que receberam orientação nutricional apresentaram menores pontuações no domínio emocional e na escala sintomas, respectivamente. Conclusão: A pontuação média global da qualidade de vida desta amostra foi satisfatória, apesar dos domínios emocional, sintomas, perspectivas futuras, imagem corporal e função sexual serem os mais afetados. O excesso de peso associou-se à piora na avaliação dos aspectos emocionais e ao agravo de sintomas.
\end{abstract}

Descritores: Neoplasias da Mama; Qualidade de Vida; Estado Nutricional.

\begin{abstract}
Objective: To assess the quality of life and its relationship with the nutritional status of women diagnosed with breast cancer. Methods: Cross-sectional study that evaluated women diagnosed with breast cancer attending a philanthropic program to fight cancer in Brusque, Santa Catarina, between April and June 2018. The exclusion criterion was: cognitive inability to answer the European Organization for Research and Treatment of Cancer questionnaires (QLQ-C30 and QLQ-BR23) or impossibility of collecting anthropometric measurements to classify nutritional status. The total scores of the questionnaires and their scales and domains were analyzed as variables. Results: 40 women participated, being 16 overweight (40\%), 14 eutrophic (35\%) and 10 obese (25\%). The mean value of the QLQ-C30 was $72.2 \pm 26.3$ points, with no difference between the categories of nutritional status. The most affected domains of the functional scale were registered as: emotional, cognitive, and symptoms scale, with insomnia, pain, and fatigue. Overweight women showed worsening in the domains of both scales. The domains' future perspectives, body image, sexual function, and arm symptoms were more compromised. Women who underwent breast reconstruction and those who received nutritional guidance had lower scores in the emotional domain and the symptoms scale, respectively. Conclusion: The mean global quality of life score of this sample was satisfactory, although the emotional, symptoms, future perspectives, body image, and sexual function domains were the most affected. Excess weight was associated with worsening in the assessment of emotional aspects and worsening of symptoms.
\end{abstract}

Descriptors: Breast Neoplasm; Quality of Life; Nutritional Status. 


\section{RESUMEN}

Objetivo: Evaluar la calidad de vida y su relación con el estado nutricional de mujeres con el diagnóstico de cáncer de mama. Métodos: Estudio transversal que evaluó mujeres con el diagnóstico de cáncer de mama del programa filantrópico de combate al cáncer de Brusque, Santa Catarina, entre abril y junio de 2018. Se tuvo como criterio de exclusión la incapacidad cognitiva para contestar a los cuestionarios European Organization for Research and Treatment of Cancer (QLQ-C30 e QLQ-BR23) o la imposibilidad de recoger datos de las medidas antropométricas para la clasificación del estado nutricional. El total de puntos de los cuestionarios y de sus escalas y dominios fueron las variables analizadas. Resultados: Participaron 40 mujeres y de ellas 16 tenían sobrepeso (40\%), 14 tenían eutrofia (35\%) y 10 tenían obesidad (25\%). El valor medio del QLQ-C30 fue de $72,2 \pm 26,3$ puntos sin diferencia entre las categorias del estado nutricional. Los dominios más afectados de la escala funcional fueron: el emocional, el cognitivo y la escala síntomas, con el insomnio, el dolor y la fatiga. Mujeres de sobrepeso presentaron empeoramiento de los dominios de ambas las escalas. Los dominios perspectivas futuras, imagen corporal, función sexual y síntomas del brazo se presentaron peor. Mujeres que han hecho la reconstrucción de las mamas y las que han recibido orientación nutricional presentaron menos puntuaciones del dominio emocional y en la escala síntomas, respectivamente. Conclusión: La puntuación media global de la calidad de vida de esa muestra ha sido satisfactoria, aunque los dominios emocional, síntomas, perspectivas futuras, imagen corporal y función sexual son los más afectados. El exceso de peso se asoció con el empeoramiento de la evaluación de los aspectos emocionales y el agravio de los síntomas.

Descriptores: Neoplasias de la Mama; Calidad de Vida; Estado Nutricional.

\section{INTRODUÇÃO}

O câncer de mama é a neoplasia com maior incidência e mortalidade na população feminina, tanto em países desenvolvidos quanto em desenvolvimento. No Brasil, corresponde a $29 \%$ dos novos casos de câncer a cada ano. No entanto, prevenção, diagnóstico precoce e tipos de tratamentos vêm prolongando a sobrevida de pacientes com câncer de mama(1).

Apesar de melhorias nas taxas de sobrevivência de pacientes com câncer de mama, principalmente em países desenvolvidos, em decorrência do aumento da conscientização do diagnóstico precoce e dos avanços nas terapias ${ }^{(2)}$, são comumente relatados, pelas pacientes, efeitos negativos do tratamento antineoplásico relacionados a aspectos físicos, emocionais e sociais com consequente redução na qualidade de vida(3).

Diferentes abordagens terapêuticas isoladas e/ou em combinação são utilizadas no tratamento de mulheres com diagnóstico de câncer de mama ${ }^{(4)}$. Fadiga e adinamia colaboram para a inatividade física, que vem acompanhada de alterações na composição corporal, como perda de massa muscular, funcionalidade e, consequentemente, perda da autonomia para realização das atividades diárias ${ }^{(2,4,5)}$. O tratamento antineoplásico também causa efeito sobre 0 estado nutricional, principalmente na presença de efeitos colaterais, como xerostomia, náuseas, vômitos, alterações na percepção do paladar e/ou olfato que resultam em inapetência ${ }^{(6,7)}$. Déficits nutricionais estão relacionados à redução da imunidade, assim como menor adesão ao tratamento e sofrimento emocional( ${ }^{(5)}$. Por outro lado, pode-se também observar ganho de peso após uso de quimioterápicos, sendo que este quando em excesso associa-se à redução de qualidade de vida e sobrevida das pacientes ${ }^{(2-4)}$.

A saúde é o maior recurso para o desenvolvimento social, econômico e pessoal, assim como uma importante dimensão da qualidade de vida, pois é entendida não como um objetivo em si, mas como um recurso fundamental para a vida cotidiana. Como um conjunto de estratégias e formas de produzir saúde, no âmbito individual e coletivo, a promoção da saúde visa atender às necessidades sociais de saúde e garantir a melhoria da qualidade de vida da população(6).

Diante desse contexto, torna-se relevante avaliar a qualidade de vida e o estado nutricional de pacientes com diagnóstico de câncer de mama, com o intuito de delinear estratégias de intervenção, sejam elas de tratamento, prevenção ou promoção da saúde, que reduzam o aparecimento de complicações precoces e tardias ao tratamento ${ }^{(6,8)}$. Dessa forma, o presente estudo tem como objetivo avaliar a qualidade de vida e sua relação com o estado nutricional de mulheres diagnosticadas com câncer de mama.

\section{MÉTODOS}

Trata-se de um estudo transversal, quantitativo e descritivo. Para fazerem parte deste estudo, selecionaram-se de forma intencional todas as 64 mulheres com diagnóstico de câncer de mama frequentadoras da Rede Feminina 
de Combate ao Câncer do município de Brusque, Santa Catarina, no período de coleta de dados realizada de abril a junho de 2018. Os critérios de inclusão adotados apresentam-se por: ter idade superior a 18 anos e aceitar participar da pesquisa mediante assinatura do Termo de Consentimento Livre e Esclarecido. Porém, incapacidade cognitiva de responder as perguntas dos questionários e impossibilidade na coleta das medidas antropométricas apresentam-se como critérios de exclusão adotados para a pesquisa.

A coleta de dados sociodemográficos das participantes (idade, estado civil e número de filhos) e clínicos (tempo de diagnóstico, presença e tempo de tratamento neoadjuvante/ adjuvante, intervalo de tempo da cirurgia até o momento da entrevista, presença de reconstrução mamária e se recebeu orientação nutricional) se deu por meio de entrevista guiada a partir de um roteiro elaborado pelos autores para este estudo. Houve também a coleta de dados antropométricos (peso atual e estatura) de todas as participantes para cálculo do índice de massa corporal (IMC). A aferição das medidas e a classificação do estado nutricional realizadas, apresentaram-se conforme a Norma Técnica do Sistema de Vigilância Alimentar e Nutricional do Ministério da Saúde ${ }^{(9)}$.

Já para avaliação da qualidade de vida, aplicaram-se dois questionários: European Organization for Research and Treatment of Cancer - Quality of Life Questionnaire (EORTC-QLQ-30) ${ }^{(10)}$ e EORTC Quality of Life QuestionnaireBreast Cancer-23 (EORTC-QLQ-BR-23)(11). A aplicação dos questionários com as participantes ocorreu de forma presencial pelo pesquisador em sala disponível exclusivamente para este fim na Rede Feminina e com tempo médio de coleta de dados de 30 minutos. Os questionários e o roteiro de entrevista, devidamente impresso e preenchido, foram identificados por números arábicos e armazenados até o preenchimento da planilha de dados.

O questionário EORTC-QLQ-30 (versão 3.0) é um instrumento traduzido para o português, que contém 30 itens, englobados em 16 domínios, e apresentados em quatro escalas: Escala de Estado de Saúde Global e Qualidade de Vida (um domínio; dois itens); Escala Funcional (cinco domínios; quinze itens); Escala de Sintomas (nove domínios; doze itens) e Escala de Dificuldades Financeiras (um domínio; um item). As questões de um a 28 do questionário são colocadas em Escala do tipo Likert de quatro pontos, onde as respostas seguem o seguinte padrão: não (valor do escore $=1$ ); pouco (valor do escore $=2$ ); moderadamente (valor do escore $=3$ ); e muito (valor do escore $=4$ ). As questões 29 e 30 apresentam-se em Escala do tipo Likert de sete pontos, onde um corresponde a péssimo e sete corresponde a ótimo. Os valores em escores variam de 0 a 100 sendo que, na Medida Global de Saúde, considerase que quanto mais o alto o escore total, mais próximo do nível saudável de qualidade de vida se encontrava a paciente. O contrário aplica-se para as escalas: Funcional, de Sintomas e de Dificuldades Financeiras, nas quais se consideraram quanto mais altos os níveis dos escores encontrados, maior o comprometimento da paciente ${ }^{(10)}$.

E o questionário EORTC-QLQ-BR 23 contém 23 questões divididas em dois grupos: Escala Funcional, que inclui quatro itens sobre imagem corporal, sendo dois itens sobre função sexual, um item sobre prazer sexual e um item sobre perspectivas futuras; Escala de Sintomas, que inclui sete itens sobre efeitos secundários da terapia sistêmica, quatro itens sobre sintomas na mama, três itens sobre sintomas no braço e um item sobre queda de cabelo. Em ambas as escalas, quanto mais alto o escore obtido maior o nível de comprometimento vivenciado pelas participantes ${ }^{(11)}$.

Todos os dados foram tabulados em uma planilha no software Microsoft Office Exce/ $(2003)$ e analisados com auxílio do programa Statistic $®$ (versão 2008). Dados sociodemográficos e clínicos apresentam-se descritos no texto em frequência relativa e absoluta, média \pm desvio-padrão ou mediana (mínimo e máximo), estando os dados dos questionários de qualidade de vida apresentados em tabelas. Verificou-se a normalidade das variáveis pelo teste Kolmogorov. Para comparação entre categorias do estado nutricional dos valores em escores obtidos dos questionários de qualidade de vida utilizou-se o teste ANOVA, com post-roc de Duncan, de acordo com a normalidade dos dados. A correlação entre as variáveis contínuas realizou-se por meio da correlação de Pearson (dados paramétricos) ou de Spearman (dados não paramétricos). Adotou-se o teste T para verificação da diferença de escores entre quem realizou reconstrução mamária e recebeu ou não orientação nutricional. Adotou-se também uma margem de erro de $5 \%$, sendo $95 \%$ o nível de confiança, nas análises estatísticas.

Esta pesquisa obteve a aprovação do Comitê de Ética em Pesquisa em Seres Humanos (CEPH) da Universidade Regional de Blumenau, sob Parecer n. ${ }^{\circ} 2.440 .498$.

\section{RESULTADOS}

Das mulheres selecionadas para fazerem parte deste estudo, 40 atenderam os critérios de inclusão e exclusão. Com idade média de $58,7 \pm 8,9$ anos, o grupo apresentou-se como sendo a maioria das mulheres casadas $(82,5 \%$; $\mathrm{n}=53$ ) e com $3 \pm 1$ filhos. O tempo mediano de diagnóstico da neoplasia de mama foi de seis anos (1 a 24 anos) e o de início do tratamento quimioterápico de três ( 1 a 18 anos). Apenas 12,5\% $(n=5)$ das entrevistadas realizaram 
reconstrução mamária e metade $(n=20)$ recebeu orientação nutricional. Quanto ao estado nutricional, a maioria apresentava sobrepeso $(40 \% ; n=16)$, seguida da categoria de eutrofia $(35 \% ; n=14)$ e obesidade $(25 \% ; n=10)$.

$\mathrm{Na}$ Tabela I apresentam-se os valores médios da pontuação dos questionários de qualidade de vida, além do valor médio da medida global de saúde do QLQ-C30 de 72,2 $\pm 26,3$ pontos, sem diferença entre as categorias de estado nutricional. Na escala funcional, apresenta-se maior valor médio para o domínio emocional (39,7 $\pm 26,8$ pontos), seguido do cognitivo $(29,1 \pm 24,3$ pontos). Nesta escala, no domínio emocional, mulheres com sobrepeso tiveram valores médios maiores do que as eutróficas. Na escala sintomas, os domínios com maiores valores médios, em ordem decrescente, são: insônia ( $43,3 \pm 31,3$ pontos); dor ( $25,4 \pm 26,1$ pontos) e fadiga ( $21,9 \pm 18,4$ pontos). Nesta escala, mulheres obesas comparadas às eutróficas demonstraram valor médio maior para os domínios dispneia e dor. No domínio fadiga, o valor médio no grupo de sobrepeso apresentou-se superior ao das mulheres com eutrofia. Não houve diferença entre as categorias do estado nutricional para a escala dificuldade financeira.

O questionário QLQ-BR23 revelou que, na escala funcional, o domínio perspectivas futuras ( $50,8 \pm 41,3$ pontos) apresentou maior pontuação média, seguido da imagem corporal $(21,4 \pm 26,4$ pontos) e da função sexual $(20,4 \pm$ 23,1 pontos). Já na escala sintomas, o maior valor médio encontrou-se no domínio referente aos sintomas no braço $(33,0 \pm 26,2$ pontos $)$.

Tabela I - Médias \pm Desvios-padrão dos escores das escalas e dos domínios dos questionários de qualidade de vida, conforme classificação do estado nutricional. Blumenau, Santa Catarina, Brasil, 2018.

\begin{tabular}{|c|c|c|c|c|c|}
\hline Variável & $\begin{array}{l}\text { Total } \\
(n=40)\end{array}$ & $\begin{array}{l}\text { Eutrofia } \\
(n=14)\end{array}$ & $\begin{array}{c}\text { Sobrepeso } \\
(n=16)\end{array}$ & $\begin{array}{l}\text { Obesidade } \\
(n=10)\end{array}$ & $p$ \\
\hline \multicolumn{6}{|l|}{ QLQ-C30 (pontos) } \\
\hline Medida Global de saúde & $72,2 \pm 26,3$ & $71,4 \pm 30,2$ & $78,1 \pm 19,9$ & $64,1 \pm 29,9$ & 0,50 \\
\hline Escala Funcional & $21,5 \pm 15,0$ & $22,8 \pm 18,3$ & $18,4 \pm 13,6$ & $24,4 \pm 12,4$ & 0,57 \\
\hline Função física & $14,6 \pm 13,1$ & $12,3 \pm 11,9$ & $15,8 \pm 13,3$ & $16,0 \pm 15,4$ & 0,78 \\
\hline Desempenho de papéis & $12,9 \pm 21,5$ & $7,14 \pm 19,2$ & $13,5 \pm 22,1$ & $20,0 \pm 23,3$ & 0,23 \\
\hline Função emocional & $39,7 \pm 26,8$ & $25,5 \pm 18,6^{a}$ & $51,0 \pm 31,7^{b}$ & $41,6 \pm 20,0^{\mathrm{ab}}$ & 0,03 \\
\hline Função cognitiva & $29,1 \pm 24,3$ & $26,1 \pm 16,9$ & $34,3 \pm 28,1$ & $25,0 \pm 27,4$ & 0,63 \\
\hline Função social & $6,66 \pm 16,7$ & $1,19 \pm 4,45$ & $10,4 \pm 23,4$ & $8,3 \pm 14,1$ & 0,34 \\
\hline Escala Sintomas & $19,7 \pm 13,4$ & $14,6 \pm 14,1$ & $19,6 \pm 11,5$ & $26,9 \pm 13,2$ & 0,08 \\
\hline Fadiga & $21,9 \pm 18,4$ & $13,4 \pm 17,5^{\mathrm{a}}$ & $25,6 \pm 13,2^{b}$ & $27,7 \pm 23,5^{\mathrm{ab}}$ & 0,03 \\
\hline Náuseas e vômitos & $10,0 \pm 15,4$ & $5,9 \pm 10,5$ & $12,5 \pm 18,7$ & $11,6 \pm 15,8$ & 0,64 \\
\hline Dor & $25,4 \pm 26,1$ & $14,2 \pm 19,4^{a}$ & $27,0 \pm 30,9^{a}$ & $38,3 \pm 20,8^{b}$ & 0,03 \\
\hline Dispneia & $15,0 \pm 24,9$ & $7,14 \pm 19,2^{\mathrm{a}}$ & $10,4 \pm 20,0^{a}$ & $33,3 \pm 31,4^{b}$ & 0,03 \\
\hline Insônia & $43,3 \pm 31,3$ & $45,2 \pm 33,6$ & $43,7 \pm 31,5$ & $40,0 \pm 30,6$ & 0,92 \\
\hline Perda de apetite & $10,8 \pm 20,5$ & $11,9 \pm 24,8$ & $8,3 \pm 14,9$ & $13,3 \pm 23,3$ & 0,91 \\
\hline Constipação & $20,0 \pm 28,0$ & $19,0 \pm 28,3$ & $12,5 \pm 20,6$ & $33,3 \pm 35,1$ & 0,24 \\
\hline Diarreia & $16,2 \pm 34,6$ & $17,8 \pm 37,2$ & $6,2 \pm 17,0$ & $30,0 \pm 48,3$ & 0,42 \\
\hline Escala dificuldade financeira & $21,6 \pm 30,7$ & $23,8 \pm 35,6$ & $22,9 \pm 29,1$ & $16,6 \pm 28,3$ & 0,83 \\
\hline \multicolumn{6}{|l|}{ QLQ-BR23 (pontos) } \\
\hline Escala Funcional & $24,2 \pm 17,1$ & $19,3 \pm 18,0$ & $26,5 \pm 17,6$ & $27,5 \pm 14,8$ & 0,41 \\
\hline Imagem Corporal & $21,4 \pm 26,4$ & $17,8 \pm 27,1$ & $24,4 \pm 28,7$ & $21,6 \pm 23,3$ & 0,56 \\
\hline Função sexual & $20,4 \pm 23,1$ & $14,2 \pm 20,5$ & $22,9 \pm 24,2$ & $25,0 \pm 25,1$ & 0,46 \\
\hline Satisfação sexual & $16,6 \pm 23,8$ & $11,9 \pm 21,1$ & $18,7 \pm 24,2$ & $20,0 \pm 28,1$ & 0,66 \\
\hline Perspectivas futuras & $50,8 \pm 41,3$ & $42,8 \pm 42,2$ & $50,0 \pm 45,5$ & $63,3 \pm 33,1$ & 0,50 \\
\hline Escala Sintomas & $23,1 \pm 13,6$ & $16,6 \pm 13,5$ & $27,2 \pm 12,1$ & $25,7 \pm 13,9$ & 0,08 \\
\hline Eventos adversos da terapia sistêmica & $20,3 \pm 12,9$ & $14,2 \pm 10,2$ & $22,9 \pm 12,4$ & $24,7 \pm 15,0$ & 0,08 \\
\hline Sintomas da mama & $21,4 \pm 17,3$ & $17,2 \pm 17,4$ & $25,0 \pm 17,7$ & $21,6 \pm 17,2$ & 0,48 \\
\hline Sintomas do braço & $33,0 \pm 26,2$ & $20,6 \pm 22,1$ & $41,6 \pm 26,7$ & $36,6 \pm 26,2$ & 0,07 \\
\hline Perda dos cabelos & $20,0 \pm 35,2$ & $19,0 \pm 38,5$ & $22,9 \pm 37,9$ & $16,6 \pm 28,3$ & 0,89 \\
\hline
\end{tabular}

Legenda: QLQ-C30: Questionário de Qualidade de Vida de 30 Itens; QLQ-BR23: Questionário de Qualidade de Vida Câncer de Mama de 23 itens; $p$ : nível de significância; *: $p<0,05$; letras diferentes nas linhas: diferença entre as categorias do estado nutricional; demais valores: demonstrados em média \pm desvio padrão 
Mulheres que realizaram reconstrução mamária apresentaram valor médio do domínio emocional (escala funcional do QLQ-C30) menor do que as que não realizaram (Figura 1). E mulheres que receberam orientação nutricional apresentaram menor valor médio atribuído ao sintoma náuseas da escala sintomas, do que as que não receberam. (Figura 2).

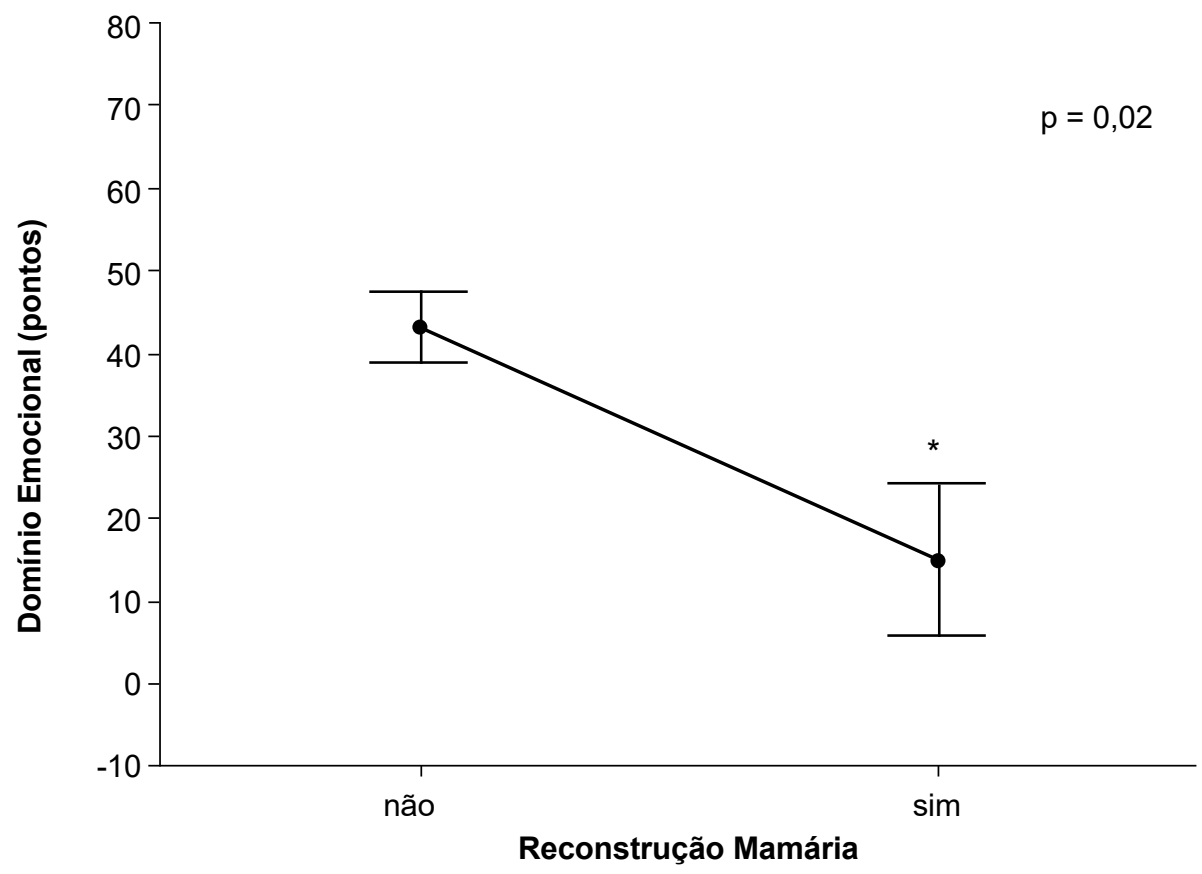

Legenda: p: nível de significância

Figura 1 - Relação entre realização de reconstrução mamária e domínio emocional da escala funcional do questionário QLQ-C30. Blumenau, Santa Catarina, Brasil, 2018.

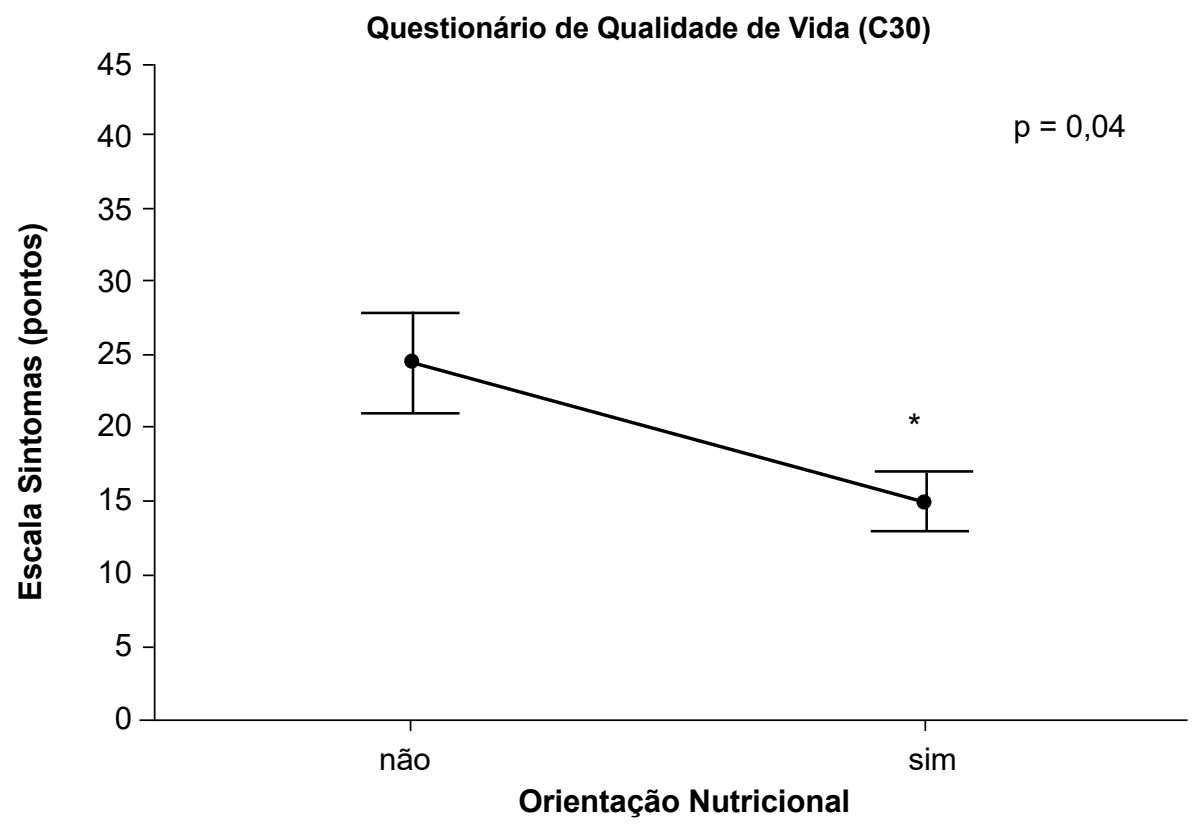

Legenda: p: nível de significância

Figura 2 - Relação entre receber orientação nutricional e pontuação da escala funcional do questionário QLQ-C30. Blumenau, Santa Catarina, Brasil, 2018.

Além disso, verificou-se correlação positiva do IMC com a pontuação total da escala sintomas dos dois questionários de qualidade de vida e com a escala funcional do QLQ-C30 (Figura 3). Encontrou-se também correlação positiva entre o número de ciclos de quimioterapia e o domínio fadiga da escala de sintomas do QLQ-C30 $(r=0,48)$ e correlação negativa entre os ciclos de quimioterapia e a pontuação da escala medida global de saúde $(r=-0,37)$ (Figura 4). 


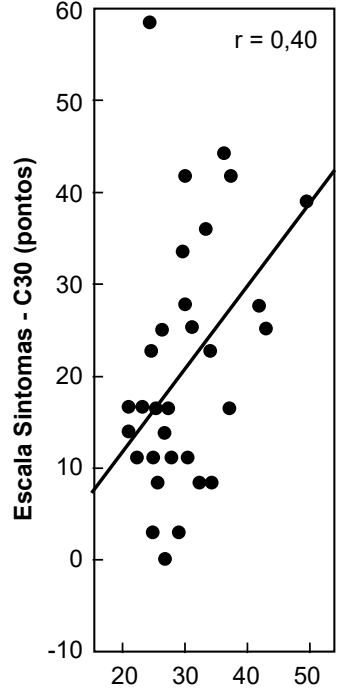

A)

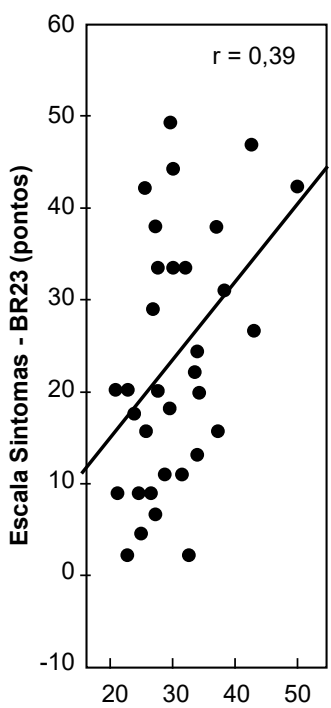

B)

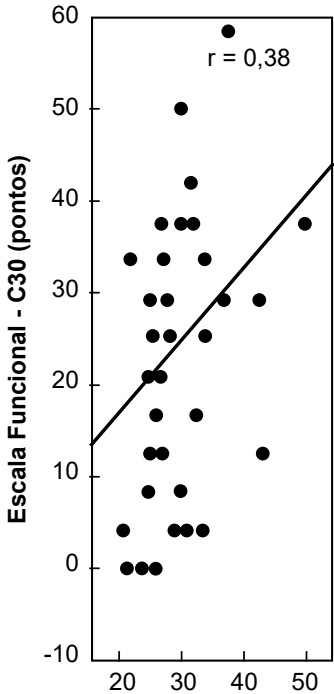

C)

Legenda: p: nível de significância

Figura 3 - Correlação entre IMC (kg/m²) e pontuação das escalas de sintomas do QLQ-C30 (A) e do BR-23 (B) e da escala funcional do QLQ-C30 (C). Blumenau, Santa Catarina, Brasil, 2018.
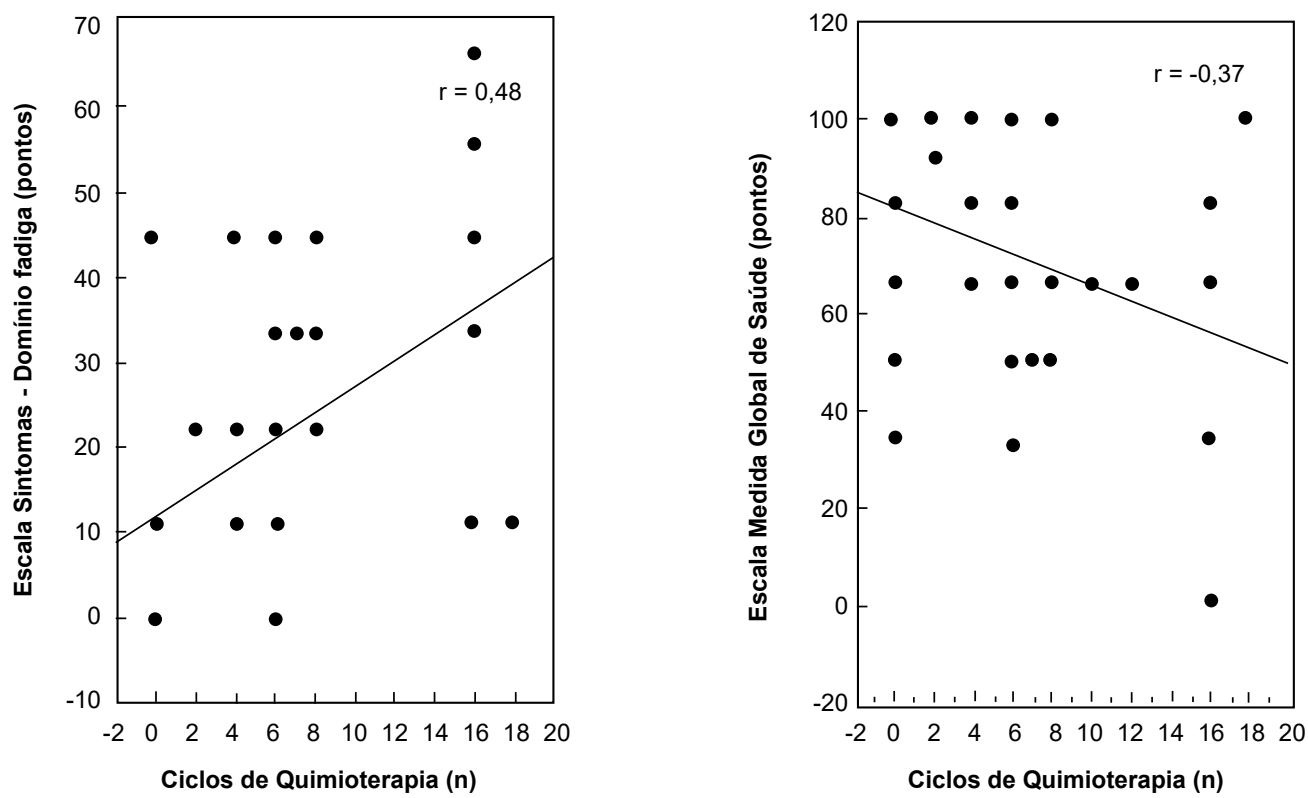

Legenda: p: nível de significância

Figura 4 - Correlação entre número de ciclos de quimioterapia e pontuação das escalas sintomas do Questionário de Qualidade de Vida (C30). Blumenau, Santa Catarina, Brasil, 2018.

\section{DISCUSSÃO}

Ao longo dos anos, o prognóstico e a taxa de sobrevivência de mulheres com câncer de mama melhoraram significativamente em todo o mundo ${ }^{(12)}$. Estudos na área da oncologia têm, cada vez mais, se proposto a avaliar a qualidade de vida de pacientes com neoplasia, uma vez que os diversos tratamentos antineoplásicos, apesar de terem acrescentados "anos à vida", podem não ter acrescentado "vida aos anos"(2). Neste sentido, vigiar, monitorar e avaliar informações sobre as condições de saúde, entre elas a qualidade de vida, de indivíduos ou grupos populacionais são estratégias para concretizar ações de promoção à saúde, respeitando valores, princípios, objetivos e diretrizes do Programa Nacional de Promoção da Saúde ${ }^{(6)}$. 
Com objetivo de avaliação e monitoramento, identificam-se estados de saúde e qualidade de vida satisfatórios, no grupo de mulheres com diagnóstico de câncer de mama que participaram deste estudo. Semelhantes resultados encontraram-se em outros estudos com pacientes com câncer de mama em tratamento antineoplásico ${ }^{(13)}$ e sem histórico de metástases ${ }^{(14)}$. No entanto, neste grupo os domínios emocional e cognitivo da escala funcional apresentaram-se mais comprometidos. Cita-se que a piora da função cognitiva e do desempenho emocional são manifestações que habitualmente ocorrem durante e após período de tratamento antineoplásico, com correlação positiva entre ambos ${ }^{(15)}$.

Além do sofrimento psicológico prolongado e dos efeitos colaterais do tratamento( ${ }^{(15)}$, acredita-se que o comprometimento dos domínios emocional e cognitivo pode estar relacionado com a idade (metade era idosa) e com o estado nutricional (dois terços apresentavam excesso de peso) das mulheres aqui avaliadas. $O$ avançar da idade e a presença de obesidade comportam-se como fatores de risco positivo para o desenvolvimento de alterações cognitivas, que tendem a se relacionar com outras comorbidades ${ }^{(16)}$. Redução e/ou perda da função cognitiva e a presença de psicopatologias podem causar declínio cognitivo, comprometer autonomia na prática das atividades diárias e prejudicar convívio social durante o envelhecimento ${ }^{(17)}$. Além disso, cita-se que o declínio da função cognitiva está relacionado aos mecanismos fisiopatológicos que determinam a obesidade ${ }^{(13-18)}$.

Neste trabalho, mulheres com sobrepeso apresentaram pior pontuação em escores do domínio emocional. Resultados similares apresentaram-se no estudo em que a maioria das participantes teve seu estado nutricional classificado como sobrepeso ${ }^{(13)}$. Possivelmente, a presença de excesso de peso prejudica aspectos emocionais relacionados à depressão e ansiedade, que isoladamente ou em conjunto, colaboram para piora da qualidade de vida $^{(19-20)}$. E, como agravante da condição nutricional, dificuldades para lidar com situações negativas da vida podem favorecer o consumo excessivo de alimentos como forma de gratificação ou como um mecanismo de defesa para diminuir angústias e tensões emocionais ${ }^{(21)}$.

Quanto à avaliação realizada com o instrumento QLQBR-23, perspectivas futuras, imagem corporal e função sexual apresentam-se como os domínios da escala funcional mais comprometidos neste estudo. Baixa expectativa de vida relaciona-se com a piora da qualidade de vida, o avanço da doença e a presença da depressão(22). Por outro lado, a função sexual pode ser afetada pelo próprio tratamento quimioterápico (com perda de lubrificação vaginal e menopausa induzida pelo medicamento) ou pela idade da paciente, que, associadas à retirada da mama, colaboram para diminuição da libido e aparecimento de problemas de autoimagem ${ }^{(23)}$. A imagem corporal, o olhar negativo para com o próprio corpo e a baixa autoestima são manifestações comuns vivenciadas por mulheres diagnosticadas com câncer de mama e estão diretamente relacionadas à retirada parcial ou total da mama, associada à perda da feminilidade ${ }^{(24,25)}$.

No presente estudo, as mulheres que não realizaram reconstrução mamária apresentaram piora do domínio emocional. Parece que mulheres que realizam reconstrução tardia da mama apresentam piora da imagem corporal quando comparadas àquelas com reconstrução imediata ${ }^{(26)}$. Mulheres submetidas à mastectomia e reconstrução também apresentam maior satisfação com a mama e bem-estar sexual, em comparação com as submetidas à cirurgia do tipo conservadora e à mastectomia total sem reconstrução ${ }^{(27)}$. Por outro lado, a prática de conservação da mama pode estar associar a uma menor satisfação com as mamas, menor bem-estar sexual e físico, principalmente devido à presença de desconfortos e dores na região, além da piora da imagem corporal(27).

$\mathrm{Na}$ escala sintomas, neste trabalho, apresentaram-se como os domínios mais comprometidos: insônia, dor (especialmente no braço) e fadiga. Presença de insônia, dor e fadiga também apresentam-se como os sintomas mais relatados por mulheres com câncer de mama avaliadas na região oeste da Arábia Saudita ${ }^{(28)}$ e por mulheres diagnosticadas com câncer de mama e submetidas à quimioterapia ${ }^{(29)}$. A insônia é um dos sintomas referido frequentemente por pacientes com câncer de mama, além de ser agravado na presença de depressão, ansiedade, dor e fadiga ${ }^{(30)}$. A fadiga pode estar relacionada a diversos fatores, dentre eles a existência de cluster de sintomas em oncologia (relacionados à presença de anemia, dor e depressão) ${ }^{(30)}$. Os piores escores na função física, dor, escala funcional e sintomas do braço apresentaram-se por pacientes submetidas à mastectomia radical, que pode ser explicado pelo prejuízo causado nos movimentos de abdução, flexão e rotação lateral do ombro em relação à amplitude de movimento e força muscular ${ }^{(31)}$.

No presente estudo, mulheres com excesso de peso também demonstraram piora dos domínios dispneia e dor, correlação positiva com a piora da condição funcional e correlação negativa com a qualidade de vida global. Em outro estudo demonstraram-se em sobreviventes de câncer de mama correlação positiva entre os valores de IMC e a pontuação em escores do questionário de qualidade de vida, principalmente devido à presença de problemas físicos e dor ${ }^{(32)}$. Segundo os autores, perdas na função física estão associadas a um aumento nos níveis de proteína C-reativa, com piora do estado inflamatório induzido pelo ganho de peso em mulheres com IMC superior a $25 \mathrm{~kg} /$ $\mathrm{m}^{2(32)}$. Em contrapartida, em estudo a respeito de qualidade de vida e estado nutricional de mulheres com câncer de mama em tratamento quimioterápico, não se observou correlação entre as variáveis(33). 
Neste estudo, encontrou-se também correlação positiva entre o número de ciclos de quimioterapia e a pontuação obtida no domínio fadiga da escala de sintomas do QLQ-C30; e correlação negativa entre os números de ciclos de quimioterapia e a pontuação da escala medida global de saúde, ambos apresentados pelo mesmo questionário. Tratamentos adjuvantes do câncer de mama, incluindo quimioterapia, cirurgia ou terapia endócrina, muitas vezes causam efeitos colaterais que alteram gravemente a qualidade de vida das pacientes ${ }^{(3)}$. Para algumas mulheres isso significa sintomas semelhantes aos da menopausa, como ondas de calor e suores noturno. Outras podem perder a qualidade do sono, diminuir funcionamento sexual e apresenrar sofrimento psicológico ${ }^{(34)}$.

Em estudo avaliando pacientes em tratamento para câncer de mama com quimioterapia, a média dos escores do questionário de qualidade de vida resultou em $38,04^{(34)}$, ou seja, a quimioterapia se relacionou com a piora da qualidade de vida durante o tratamento, principalmente pela presença de dor, fadiga e alterações emocionais ${ }^{(15)}$. Por outro lado, os sintomas se tornam menos frequentes e intensos após o término do tratamento e a qualidade de vida tende a melhorar, exceto quando sintomas relacionados à fadiga, depressão e dor aumentam no pós-tratamento e, inevitavelmente, acabam mantendo piores os valores relacionados à qualidade de vida global(15).

Durante o tratamento quimioterápico de mulheres com câncer de mama é comum também ocorrerem alterações na mucosa do trato gastrointestinal e dificuldades na mastigação e deglutição, além de náusea e vômitos pré e pós-prandiais ${ }^{(35)}$. Sintomas que influenciam negativamente no estado nutricional e na qualidade de vida da paciente, particularmente nos aspectos relacionados às características físicas e emocionais ${ }^{(36)}$. Assim sendo, a avaliação e o acompanhamento nutricional periódico são necessários para orientar a paciente a realização de ações que tragam maior tolerância ao tratamento antineoplásico e menor risco de complicações, além de auxiliarem a prevenir ou tratar deficiências nutricionais, que prejudicam a condição de saúde e a qualidade de vida(2).

No presente estudo, pacientes que receberam orientação nutricional relataram menor presença de náuseas e vômitos. Da mesma maneira, intervenção nutricional durante os três primeiros ciclos de quimioterapia neoadjuvante em mulheres com câncer de mama apresentou-se como capaz de preservar a qualidade de vida, diminuindo a ocorrência de náuseas, vômitos e perda de apetite durante o tratamento. Esses achados reforçam a importância das intervenções nutricionais durante os tratamentos quimioterápicos a fim de minimizar possíveis impactos nutricionais, consequentes involuções clínicas durante a terapia ou mesmo a interrupção do tratamento ${ }^{(35)}$.

Monitorar o estado nutricional de mulheres diagnosticadas com câncer de mama pode ser uma estratégia de ação dentro da proposta de promoção da saúde, que objetiva melhorar a curto e longo prazo à qualidade de vida relacionada aos sintomas emocionais e a presença de fadiga, dispneia e dor, principalmente no braço. Além disso, a garantia do suporte de equipe multiprofissional a todas as mulheres antes e após o diagnóstico é importante para melhora do bem-estar e tratamento de efeitos adversos decorrentes dos tratamentos. Do ponto de vista clínico, uma das formas de melhorar a qualidade de vida das sobreviventes do câncer de mama é manter durante todo o tratamento intervenções relacionadas aos aspectos físicos e psicossociais ${ }^{(37)}$. Programas multidimensionais têm ganhado ênfase cada vez maior no cuidado integral em saúde de mulheres com diagnóstico de câncer de mama a fim de maximizar a qualidade de vida destas durante e após o tratamento da doença ${ }^{(12)}$.

Dentre as limitações encontradas no presente estudo, destaca-se a interpretação das pacientes quanto ao significado da palavra "qualidade de vida", requisito importante no tratamento de doenças crônicas, porém, subjetivo, o que carece de um acompanhamento mais detalhado de cada paciente. Por fim, outra limitação se refere ao reduzido número de participantes da pesquisa, a ampla variação no número de ciclos de quimioterapia e tempo de tratamento entre as mulheres aqui avaliadas.

\section{CONCLUSÃO}

Conclui-se que, neste grupo de mulheres com diagnóstico de câncer de mama, a qualidade de vida global considerou-se satisfatória. No entanto, dimensões relacionadas aos aspectos emocionais e funcionais apresentaramse como sendo as mais comprometidas, com destaque a presença de dor, insônia e fadiga, além das dimensões que abordavam perspectivas futuras, imagem corporal e função sexual. O excesso de peso nas mulheres avaliadas associou-se à piora na avaliação dos aspectos emocionais e ao agravo de sintomas de dispneia, fadiga e dor, principalmente no braço.

\section{CONFLITOS DE INTERESSE}

As autoras afirmam que não houve conflitos de interesse na execução desta pesquisa. 


\section{CONTRIBUIÇÕES}

Sabrina Hodecker contribuiu com a concepção e delineamento do estudo, interpretação dos resultados e a redação do conteúdo do manuscrito. Luciane Coutinho de Azevedo contribuiu com a concepção e delineamento do estudo, a análise dos dados e a revisão crítica do conteúdo do manuscrito. Todos os autores aprovaram a versão final do manuscrito e são responsáveis por todos seus aspectos, incluindo a garantia de sua precisão e integridade.

\section{FONTE DE FINANCIAMENTO}

Programa Institucional de Bolsas de Iniciação Científica (PIBIC)/ Conselho Nacional de Desenvolvimento Científico e Tecnológico (CNPq).

\section{REFERÊNCIAS}

1. Ministério da Saúde (BR); Instituto Nacional de Câncer José Alencar Gomes da Silva. Estimativa 2018Incidência de câncer no Brasil [Internet]. Rio de Janeiro: INCA; 2017 [acesso em 2021 Maio 25]. Disponível em: https://www.inca.gov.br/sites/ufu.sti.inca.local/files//media/document//estimativa-incidencia-de-cancer-nobrasil-2018.pdf

2. Shin KNL, Mun CY, Shariff ZM. Nutrition Indicators, Physical Function, and Health-Related Quality of Life in Breast Cancer Patients. Asian Pac J Cancer Prev [Internet]. 2020 [acesso em 2021 Maio 25];21(7):1939-50. Disponível em: https://pubmed.ncbi.nlm.nih.gov/32711419/

3. Dang CC, Wong KZ, Lim M, Zulkefle N. Health Related Quality of Life (HRQoL) among Breast Cancer Patients Receiving Chemotherapy in Hospital Melaka: Single Centre Experience. Asian Pac J Cancer Prev [Internet]. 2016 [acesso em 2021 Maio 25];17:5121-6. Disponível em: https://pubmed.ncbi.nlm.nih. gov/28122444/

4. De Cicco P, Catani MV, Gasperi V, Sibilano M, Quaglietta M, Savini I. Nutrition and Breast Cancer: a Literature Review on Prevention, Treatment and Recurrence. Nutrients [Internet]. 2019 [acesso em 2021 Maio 25];11(7):1514. Disponível em:https://pubmed.ncbi.nlm.nih.gov/31277273/

5. De Vries YC, Boesveldt S, Kelfkens CS, Posthuma EE, Van den Berg MMGA, Kruif JTCM, et al. Taste and smell perception and quality of life during and after systemic therapy for breast cancer. Breast Cancer Res Treat [Internet]. 2018 [acesso em 2021 Maio 25];170:27-34. Disponível em: https://pubmed.ncbi.nlm.nih. gov/29476290/

6. Ministério da Saúde (BR), Secretaria de Vigilância em Saúde, Secretaria de Atenção à Saúde. Política Nacional de Promoção da Saúde: PNPS: Anexo I da Portaria de Consolidação $n^{\circ}$ 2, de 28 de setembro de 2017, que consolida as normas sobre as políticas nacionais de saúde do SUS. Brasília: Ministério da Saúde; 2018.

7. Buch K, Gunmalm V, Andersson M, Schwarz P, Brøns C. Effect of chemotherapy and aromatase inhibitors in the adjuvant treatment of breast cancer on glucose and insulin metabolism-A sistemática review. Cancer Med [Internet]. 2019 [acesso em 2021 Maio 25];8:238-45. Disponível em: https://pubmed.ncbi.nlm.nih. gov/30561133/

8. Sanz EA, Abilés J, Siles GM, Ruíz EP, García JA, Domínguez AR. Impact of weight loss on cancer patients' quality of life at the beginning of the chemotherapy. Support Care Cancer [Internet]. 2021 [acesso em 2021 Maio 25];29:627-34. Disponível em: https://pubmed.ncbi.nlm.nih.gov/32424642/

9. Ministério da Saúde (BR), Secretaria de Atenção à Saúde, Departamento de Atenção Básica. Orientações para a coleta e análise de dados antropométricos em serviços de saúde: Norma Técnica do Sistema de Vigilância Alimentar e Nutricional - SISVAN. Brasília: Ministério da Saúde; 2011.

10. Aaronson NK, Ahmedzai S, Bergman B, Bullinger M, Cull A, Duez NJ, et al. The European Organization for Research and Treatment of Cancer QLQ-C30: a quality of life instrument for use in international clinical trials in oncology. J Natl Cancer Inst [Internet]. 1993 [acesso em 2019 Abr 15];85:365-76. Disponível em: https:// www.ncbi.nlm.nih.gov/pubmed/8433390

11. Michels FAS, Latorre MRDO, Maciel MS. Validity, reliability and understanding of the EORTC-C30 and EORTC-BR23, quality of life questionnaires specific for breast cancer. Rev Bras Epidemiol [Internet]. 2013 
[acesso em 2019 Abr 15];16(2):352-63. Disponível em: http://www.scielo.br/pdf/rbepid/v16n2/1415-790Xrbepid-16-02-00352.pdf

12. Cheng KKF, Lim YTE, Koh ZM, Tam WWS. Home-based multidimensional survivorship programmes for breast cancer survivors. Cochrane Database Syst Rev [Internet]. 2017 [acesso em 2021 Maio 25];8(8). Disponível em: https://pubmed.ncbi.nlm.nih.gov/28836379/

13. Lu Shin KN, Mun CY, Shariff ZM. Indicadores de nutrição, função física e qualidade de vida relacionada à saúde em pacientes com câncer de mama. Asian Pac J Cancer Prev [Internet]. 2020 [acesso em 2021 Maio 25];21(7):1939-50. Disponível em: https://www.ncbi.nlm.nih.gov/pmc/articles/PMC7573431/

14. Costa WA, Eleutério J Jr, Giraldo PC, Gonçalves AK. Quality of life in breast cancer survivors. Rev Assoc Med Bras [Internet]. 2017 [acesso em 2021 Maio 25];63(7):583-9. Disponível em:https://www.scielo.br/j/ramb/a/Fk FsVzNwHNxNyhcp75Rd85f/?lang=en

15. Binotto M, Schwartsmann G. Qualidade de Vida Relacionada à Saúde de Pacientes com Câncer de Mama: Revisão Integrativa da Literatura. Rev Bras Cancerol [Internet]. 2020 [acesso em 2021 Maio 25];66(1):e06405. Disponível em:https://rbc.inca.gov.br/revista/index.php/revista/article/view/405

16. Romera HP. Influência da obesidade sobre a função cognitiva de mulheres pós-menopáusicas [dissertação] [Internet]. Brasília: Universidade Católica de Brasília; 2018 [acesso em 2021 Maio 25]. Disponível em: https:// bdtd.ucb.br:8443/jspui/bitstream/123456789/1248/1/Dissertacaofinalizada.pdf

17. Konflanz F, Costa K, Mendes T. A neuropsicologia do envelhecer: as "faltas" e "falhas" do cérebro e do processo cognitivo que podem surgir na velhice. Psicologia.pt [Internet]. 2017 [acesso em 2021 Maio 25];13(8):1-6. Disponível em: https://www.psicologia.pt/artigos/textos/A1103.pdf

18. O'Brien PD, Hinder LM, Callaghan BC, Feldman EL. Consequências neurológicas da obesidade. Lancet Neurol [Internet]. 2017 [acesso em 2021 Maio 25];16:465-77. Disponível em: https://www.ncbi.nlm.nih.gov/ pmc/articles/PMC5657398/

19. Vasques F, Martins F, Azevedo A. Aspectos psiquiátricos do tratamento da obesidade. Arch Clin Psychiatry [Internet]. 2004 [acesso em 2021 Maio 27];31(4):195-8. Disponível em: https://www.scielo.br/j/rpc/ a/3ytf9qn3PYx3HftRSwyng6q/abstract/?lang=pt

20. Paffenbarger RS, Wing AL, Hyde RT. Physical activity and incidence of hypertension in college alumni. Am J Epidemiol [Internet]. 1983 [acesso em 2021 Maio 27];117:245-57. Disponível em: https://pubmed.ncbi.nlm.nih. gov/6829553/

21. Franques ARM, Arenales-Loli MS. Contribuições da psicologia na cirurgia da obesidade. São Paulo: Vetor Editora; 2006.

22. Batchelor D. Hair and cancer chemotherapy: consequences and nursing care - a literature study. Eur J Cancer Care [Internet]. 2001 [acesso em 2019 Abr 15];10(3):147-63. Disponível em: https://onlinelibrary.wiley. com/doi/full/10.1046/j.13652354.2001.00272.x?sid=nlm\%3Apubmed

23. Zee B, Huang C, Mak S, Wong J, Chan E, Yeo W. Factors related to sexual health in Chinese women with breast cancer in Hong Kong. Asia Pac J Clin Oncol [Internet]. 2008 [acesso em 2019 Abr 15];4(4):218-26. Disponível em: https://onlinelibrary.wiley.com/doi/full/10.1111/j.1743-7563.2008.00214.x

24. Juver J, Verçosa N. Depressão em pacientes com dor no câncer avançado. Rev Bras Anestesiol [Internet]. 2008 [acesso em 2019 Abr 15];58(3):287-98. Disponível em: http://www.scielo.br/pdf/rba/v58n3/en_12.pdf

25. Lagendijk M, van Egdom LSE, van Veen FEE, Vos EL, Mureau MAM, van Leeuwen N, et al. Patient-Reported Outcome Measures May Add Value in Breast Cancer Surgery. Ann Surg Oncol [Internet]. 2018 [acesso em 2019 Abr 15];(12):3563-71.Disponível em: https://pubmed.ncbi.nlm.nih.gov/30178391/

26. Teo I, Reece GP, Christie IC, Guindani M, Markey MK, Heinberg LJ, et al. Body image and quality of life of breast cancer patients: Influence of timing and stage of breast reconstruction. Psycho-Oncol [Internet]. 2016 [acesso em 2021 Maio 27];25:1106-12. Disponível em: https://www.ncbi.nlm.nih.gov/pmc/articles/PMC4988938/

27. Howes BH, Watson DI, Xu C, Fosh B, Canepa M, Dean NR. Quality of life following total mastectomy with and without reconstruction versus breast-conserving surgery for breast cancer: A case-controlled cohort study. J Plast Reconstr Aesthet Surg [Internet]. 2016 [acesso em 2021 Abr 24];69(9):1184-91. Disponível em: https:// pubmed.ncbi.nlm.nih.gov/27406255/ 
28. Imran M, Al-Wassia R, Alkhayyat SS, Baig M, Al-Saati BA. Assessment of quality of life (QoL) in breast cancer patients by using EORTC QLQ-C30 and BR-23 questionnaires: a tertiary care center survey in the western region of Saudi Arabia. Plos One [Internet]. 2019 [acesso em 2021 Maio 26];14(7). Disponível em: https:// pubmed.ncbi.nlm.nih.gov/31291302/

29. Charalambous A, Kaite CP, Charalambous M, Tistsi T, Kouta C. The effects on anxiety and quality of life of breast cancer patients following completion of the first cycle of chemotherapy. J Sage Open Med [Internet]. 2017 [acesso em 2019 Abr 15];5:1-10. Disponível em: https://www.ncbi.nlm.nih.gov/pmc/articles/ PMC5495504/

30. Gehrman P, Garland S, Matura L, Mao J. Insomnia in breast cancer: Independent symptom or symptom cluster?. Palliat Support Care [Internet]. 2016 [acesso em 2019 Abr 15];15(3):369-75. Disponível em: https:// www.ncbi.nlm.nih.gov/pubmed/27917746

31. Boing L, Araujo C, Pereira G, Moratelli J, Benneti M, Borgatto A, et al. Tempo sentado, imagem corporal e qualidade de vida em mulheres após a cirurgia do câncer de mama. Rev Bras Med Esporte [Internet]. 2017 [acesso em 2019 Abr 15];23(5):366-70. Disponível em: http://www.scielo.br/pdf/rbme/v23n5/1517-8692rbme-23-05-00366.pdf

32. Imayama I, Alfano CM, Neuhouser ML, George SM, Smith WA, Baumgartner RN, et al. Weight, inflammation, cancer-related symptoms and heath related quality of life among breast cancer survivors. Breast Cancer Res Treat [Internet]. 2013 [acesso em 2019 Abr 15];140(1):159-76. Disponível em: https://www.ncbi.nlm.nih.gov/ pmc/articles/PMC3772666/

33. Scheibler J, Silva FMM, Moreira TR, Adami FS. Qualidade de vida, estado nutricional e consumo alimentar de mulheres com câncer de mama em tratamento quimioterápico. Rev Bras Promoç Saúde [Internet]. 2016 [acesso em 2021 Maio 25];(4):544-53. Disponível em: https://periodicos.unifor.br/RBPS/article/view/4421/pdf

34. Bray F, Ferlay J, Soerjomataram I, Siegel RL, Torre LA, Jemal A. Global cancer statistics 2018: GLOBOCAN estimates of incidence and mortality worldwide for 36 cancers in 185 countries. Cancer J Clin [Internet]. 2018 [acesso em 2021 Maio 25];68(6):394-424. Disponível em: https://pubmed.ncbi.nlm.nih.gov/30207593/

35. Souza APS, Silva LC, Fayh APT. Nutritional Intervention Contributes to the Improvement of Symptoms Related to Quality of Life in Breast Cancer Patients Undergoing Neoadjuvant Chemotherapy: A Randomized Clinical Trial. Nutrients [Internet]. 2021 [acesso em 2021 Maio 25];13(2):589. Disponível em: https://www.ncbi. nlm.nih.gov/pmc/articles/PMC7916763/

36. Arends J, Baracos V, Bertz H, Bozzetti F, Calder PC, Deutz NEP, et al. Recomendações do grupo de especialistas ESPEN para ação contra a desnutrição relacionada ao câncer. Clin Nutr. 2017;36:1187-96.

37. Xia J, Tang Z, Deng Q, Yang R, Wang J, Yu J. Predictors of the quality of life in Chinese breast cancer survivors. Breast Cancer Res Treat [Internet]. 2018 [acesso em 2021 Maio 26];167(2):537-45. Disponível em: https://pubmed.ncbi.nlm.nih.gov/28965272/

\section{Endereço do primeiro autor:}

Sabrina Hodecker

Rua: Luiz Gandolfi, 1702

Bairro: São Pedro

CEP: 88360-000 - Guabiruba - SC - Brasil

E-mail: sabhodecker@gmail.com

\section{Endereço para correspondência:}

Luciane Coutinho de Azevedo

Rua: São Paulo, 2171

Bairro: Itoupava Seca

CEP: 89030-001 - Blumenau - SC - Brasil

E-mail: lucianec@furb.br

Como citar: Hodecker S, Azevedo LC. Qualidade de vida e estado nutricional de pacientes diagnosticadas com câncer de mama. Rev Bras Promoç Saúde. 2021;34:11312. 